\title{
Spin-Sensitive Bleaching and Monopolar Spin Orientation in Quantum Wells
}

\author{
S. D. Ganichev, ${ }^{1,2}$ S. N. Danilov, ${ }^{1}$ V. V. Bel'kov, ${ }^{2}$ E. L. Ivchenko, ${ }^{2}$ M. Bichler, ${ }^{3}$ W. Wegscheider,,${ }^{1,3}$ \\ D. Weiss, ${ }^{1}$ and W. Prett ${ }^{1}$ \\ ${ }^{1}$ Institut für Experimentelle und Angewandte Physik, Universität Regensburg, D-93040 Regensburg, Germany \\ ${ }^{2}$ Ioffe Physico-Technical Institute, RAS, 194021, St. Petersburg, Russia \\ ${ }^{3}$ Walter Schottky Institut, TU München, 85748, Garching, Germany
}

(Received 13 July 2001; published 15 January 2002)

\begin{abstract}
Spin-sensitive bleaching of the absorption of far-infrared radiation has been observed in $p$-type GaAs/AlGaAs quantum well structures. The absorption of circularly polarized radiation saturates at lower intensities than that of linearly polarized light due to monopolar spin orientation in the first heavy-hole subband. Spin relaxation times of holes in $p$-type material in the range of tens of ps were derived from the intensity dependence of the absorption.
\end{abstract}

DOI: 10.1103/PhysRevLett.88.057401

The spin of electrons and holes in solid state systems is the decisive ingredient for active spintronic devices [1,2] and several schemes of quantum computation [3-5]. Especially the combination of ferromagnetic materials with semiconductors seems to be a promising combination for novel functional concepts. Open problems which have to be addressed in this respect involve spin injection into semiconductors, spin relaxation in low-dimensional semiconductor structures, as well as spin detection. Significant progress was made recently: it was shown that spin polarized electrons (or holes) can be injected from magnetic semiconductor materials into semiconductors $[6,7]$. The presence of spin polarized electrons can be probed by analyzing the Kerr effect [8] or by analyzing the degree of circular polarization of light which gets emitted when polarized electrons recombine with holes. The inverse process, exciting free carriers by circularly polarized light [9], is frequently used to prepare an ensemble of spin polarized carriers. In low-dimensional systems with band splitting in $\boldsymbol{k}$ space due to $\boldsymbol{k}$-linear terms in the Hamiltonian optical excitation not only leads to a spin polarized ensemble of electrons but also to a current whose sign and magnitude depend on the degree of circular polarization of the incident light (circular photogalvanic effect [10]).

For the realization of spintronic devices long spin dephasing times in quantum well $(\mathrm{QW})$ structures are crucially needed. Spin transport must occur without destroying the relevant spin information. Current investigations of the spin lifetime in semiconductors [11-16] are based on optical spin orientation by interband excitation. Studies of such bipolar spin orientation, where both electrons and holes got excited, gave important insights into the mechanisms of spin relaxation. We show below that by combining the circular photogalvanic effect (CPGE) [10] with saturation (bleaching of absorption) spectroscopy [17-21] we are able to probe spin relaxation for monopolar spin orientation. In contrast to the conventional methods of optical spin orientation, in our measurements only one type of charge carriers (electrons or holes) gets spin oriented
PACS numbers: 78.30.Fs, 68.65.-k, 73.50.Mx, 73.50.Pz

and is involved in relaxation processes. This is achieved by using terahertz radiation which excites intraband or intersubband, but no interband, transitions. Monopolar spin orientation allows us to study spin relaxation without electron-hole interaction and exciton formation. The most important advantage of monopolar spin orientation is that relaxation processes can be investigated for electrons in $n$-type material and for holes in $p$-type material. These conditions have not been met previously in quantum wells where, due to interband excitation, only the spin relaxation times of optically generated minority carriers were accessible (for reviews, see [14-16]).

Here we report the first observation of spin-sensitive bleaching of the heavy-hole $h h 1$-light-hole $l h 1$ absorption in $p$-type $\mathrm{QW}$ structures which allows us to investigate spin relaxation for a monopolar spin orientation. The basic physics is sketched in Fig. 1. Exciting with circularly polarized light results in direct intersubband transitions at $k \neq 0$ (solid arrow) which depopulate and populate selectively spin states in the valence subbands ( $h h 1$ and $l h 1$ ) and cause a monopolar spin polarization. Spin relaxation inside the $h h 1$ subband is characterized by the relaxation time $\tau_{s}$. Relaxation from the $l h 1$ subband back to $h h 1$ is mediated by phonons and characterized by a spin independent energy relaxation time $\tau_{e}$ (broken arrows in Fig. 1). The absorption coefficient $\alpha$ is proportional to the difference of the populations (occupation numbers) of the initial and final states involved in the direct transitions. At high intensities the absorption coefficient decreases since the photoexcitation rate becomes comparable to the nonradiative relaxation rate into the initial state. Thus absorption bleaching of circularly polarized radiation is characterized by the spin relaxation time $\tau_{s}$ and the energy relaxation time $\tau_{e}$, respectively. In contrast to circularly polarized light, optical transitions induced by linearly polarized light are not spin selective and saturation is controlled by energy relaxation of photoexcited carriers only. The difference in absorption bleaching for circularly and linearly polarized radiation can be observed experimentally. 


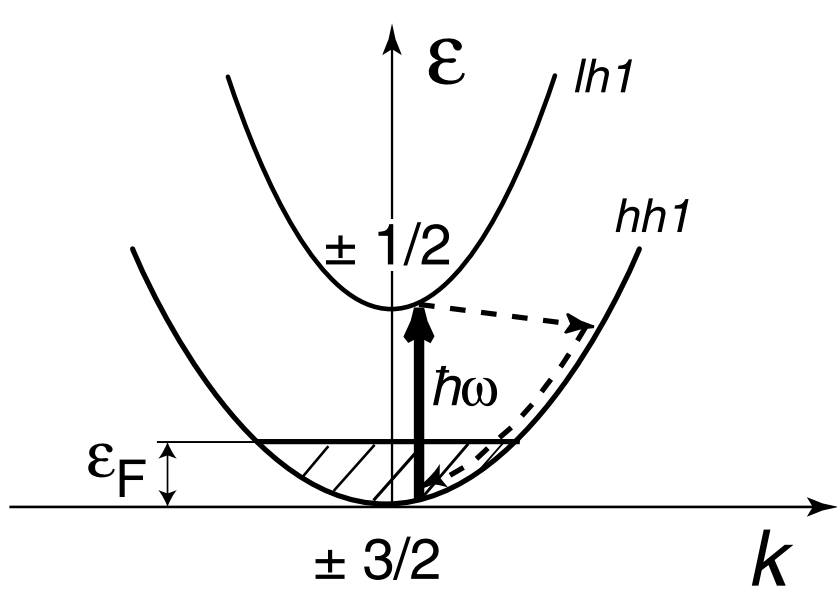

FIG. 1. Sketch of direct optical transitions (full line) between the first heavy-hole and the first light-hole subband in $p$-GaAs/AlGaAs QWs. While the splitting of the bands in $k$ space is necessary for an understanding of the circular photogalvanic effect [10], it is unimportant for the saturation process and ignored in the sketch. The absorption of far-infrared radiation with photon energy $\hbar \omega=8.3 \mathrm{meV}$ occurs very close to $k=0$. Hence, initial and final states are characterized by quantum numbers $m= \pm 3 / 2$ and $\pm 1 / 2$, respectively. Dashed lines show the energy relaxation of photoexcited carriers. $\varepsilon_{F}$ is the Fermi energy.

The experiments have been carried out on modulated doped $p$-GaAs/AlGaAs (311)-molecular-beam-epitaxy (MBE)-grown samples with a single QW or $20 \mathrm{QWs}$ of width $L_{W}=15 \mathrm{~nm}$ and with period $d=130 \mathrm{~nm}$ for multiple QW structures. Samples with free carrier densities $p_{s}$ of about $2 \times 10^{11} \mathrm{~cm}^{-2}$ and mobilities around $5 \times 10^{5} \mathrm{~cm}^{2} / \mathrm{V} \mathrm{s}$ were studied in the temperature range from 4.2 to $300 \mathrm{~K}$. A pair of Ohmic contacts was centered on opposite sample edges along the direction $x \|[1 \overline{1} 0]$ (see Fig. 2 inset). A high power far-infrared molecular laser, optically pumped by a transversely excited atmospheric pressure $\mathrm{CO}_{2}$ laser, has been used to deliver 100-ns pulses with intensities up to $1 \mathrm{MW} / \mathrm{cm}^{2}$ at wavelength $\lambda=148 \mu \mathrm{m}$. The radiation induces direct optical transitions between the first heavy-hole and the first light-hole valence subband (see Fig. 1). A $\lambda / 4$ plate has been used to obtain from the initially linearly polarized laser light circularly polarized radiation. The degree of circular polarization $P_{\text {circ }}$ was \pm 1 for right- and left-handed circularly polarized light, respectively.

The absorption of terahertz radiation by free carriers in QWs is weak and difficult to determine in transmission measurements. This is even worse in the case of bleaching at high power levels. Therefore the nonlinear behavior of the absorption has been investigated employing the recently observed circular and linear photogalvanic effects $[10,22]$. Both CPGE and the linear photogalvanic effect (LPGE) yield an easily measurable electric current in the $x$ direction [23]. The absorption coefficient is proportional to the photogalvanic current $j_{x}$ normalized by the radiation

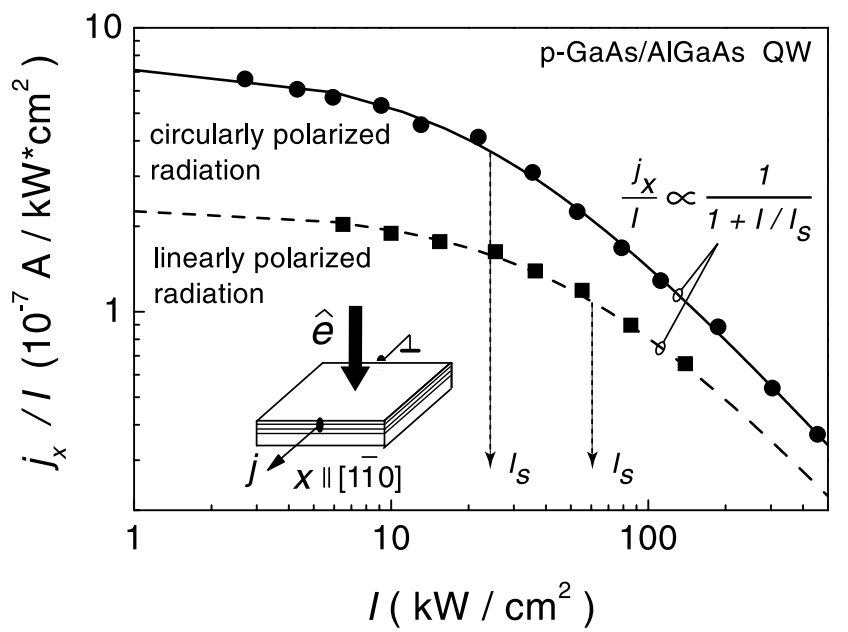

FIG. 2. Photogalvanic current $j_{x}$ normalized by the intensity $I$ as a function of $I$ for circularly and linearly polarized radiation at $T=20 \mathrm{~K}$. The inset shows the geometry of the experiment; $\hat{\boldsymbol{e}}$ indicates the direction of the incoming light. The current $j_{x}$ flows along the $[1 \overline{1} 0]$ direction at normal incidence of radiation on $p$-type (113) $A$-grown GaAs/AlGaAs QWs. In order to obtain the CPGE right or left circularly polarized light has been applied. To obtain the LPGE linearly polarized radiation with the electric field vector $E$ oriented at $45^{\circ}$ to the $x$ direction was used. The measurements are fitted to $j_{x} / I \propto 1 /\left(I+I / I_{s}\right)$ with one parameter $I_{s}$ for each state of polarization (full line: circular; broken line: linear).

intensity $I$ [24]. Thus by choosing polarization we obtain a photoresponse corresponding to the absorption coefficient of circularly or linearly polarized radiation, respectively.

Our measurements (Fig. 2) indicate that the photocurrent $j_{x}$ at a low power level depends linearly on the light intensity and gradually saturates with increasing intensity, $j_{x} \propto I /\left(1+I / I_{s}\right)$, where $I_{s}$ is the saturation intensity. This corresponds to a constant absorption coefficient at low power levels and decreasing absorption with rising intensity. Saturation intensities $I_{s}$ have been measured for temperatures between 4.2 and $200 \mathrm{~K}$. The key result is plotted in Fig. 3 and shows that the saturation intensity $I_{S}$ is generally smaller for circularly polarized radiation. The experimental values increase from about $10 \mathrm{~kW} / \mathrm{cm}^{2}$ at $4.2 \mathrm{~K}$ to $300 \mathrm{~kW} / \mathrm{cm}^{2}$ at $200 \mathrm{~K}$. At room temperature $I_{s}$ get nonmeasurably large.

Over the whole temperature range the holes occupy, in equilibrium, the lowest heavy-hole subband $h h 1$. Absorption of $\lambda=148-\mu$ m radiation ( $\hbar \omega=8.3 \mathrm{meV}$ ) occurs by direct optical transitions from $h h 1$ to the first light-hole subband $l h 1$ close to $k=0$ (but $\neq 0$ ) [25], as is sketched in Fig. 1. Thus, the selection rules for the absorption are $\Delta m= \pm 1$ with angular momentum quantum number $m= \pm 3 / 2$ for the initial and $m= \pm 1 / 2$ for the final state [26]. The insets in Fig. 3 show the corresponding transitions for linear (top left) and circular (bottom right) polarization by full arrows. Linearly polarized radiation has been decomposed in right- and left-handed circularly 


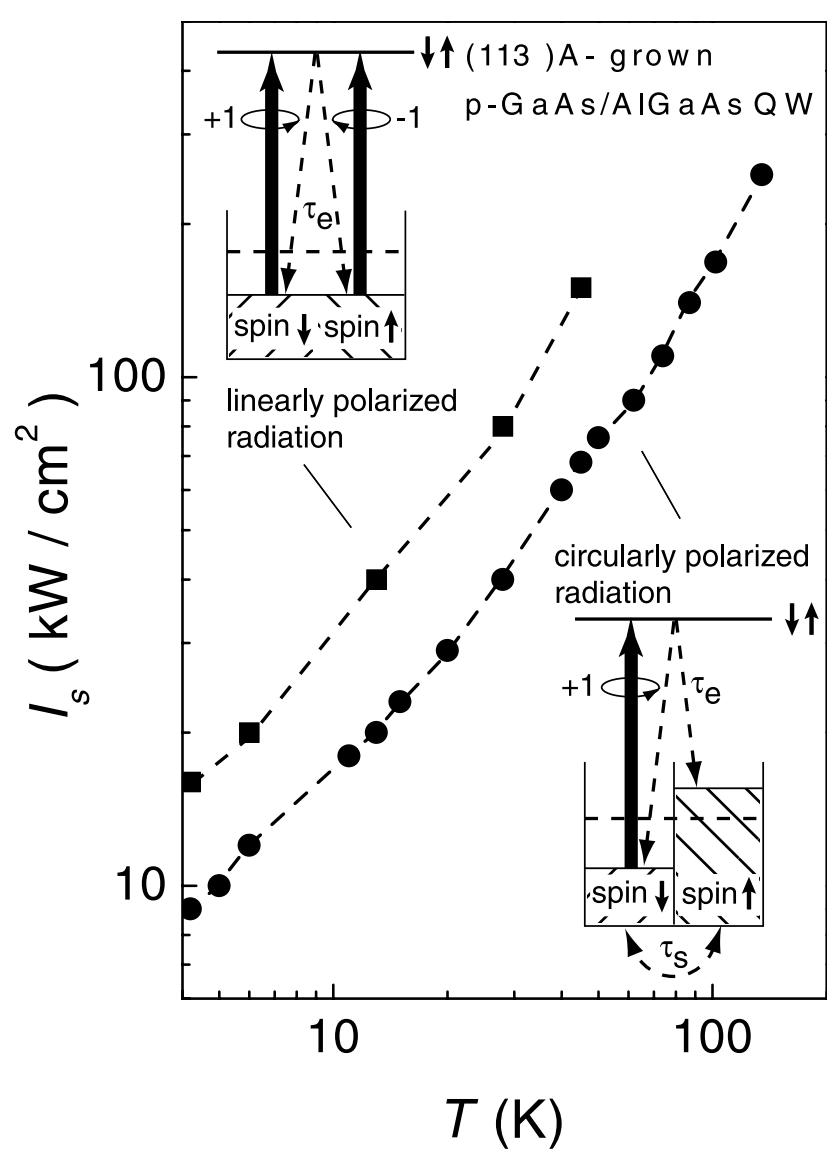

FIG. 3. Temperature dependence of the saturation intensity $I_{s}$ for linearly and circularly polarized radiation. The dependence is shown for one $p$-GaAs/AlGaAs (311)-MBE-grown sample with a single $\mathrm{QW}$ of $L_{W}=15-\mathrm{nm}$ width. The free carrier density is $1.66 \times 10^{11} \mathrm{~cm}^{-2}$ and the mobility is $6.5 \times 10^{5} \mathrm{~cm}^{2} / \mathrm{V} \mathrm{s}$. The insets show a microscopic picture explaining the origin of the difference in saturation intensities.

polarized light of identical amplitudes. Broken lines in these insets indicate nonradiative energy and spin relaxation transitions.

Linearly polarized radiation (top left inset in Fig. 3) equally depopulates spin-up and spin-down states of $h h 1$ and populates $l h 1$. With rising intensity these nonequilibrium populations approach each other causing the bleaching of absorption, controlled by the energy relaxation time $\tau_{e}$. In contrast to linear polarization the absorption of circularly polarized light is spin selective because only one type of spin is involved in absorption (right bottom inset of Fig. 3). During energy relaxation to the initial state in $h h 1$ the holes loose their photoinduced orientation due to rapid relaxation [26]. Thus, spin orientation occurs in the initial subband $h h 1$, only. Bleaching of absorption is hence controlled by two time constants, the $\tau_{e}$ and $\tau_{s}$. Note that $\tau_{e}$ is the same for circular and linear polarization. If $\tau_{s}$ is of the order of $\tau_{e}$ or larger, bleaching of absorption becomes spin sensitive and the saturation intensity of circularly polarized radiation drops below the value of linear polarization.
Spin-sensitive bleaching can be analyzed in terms of excitation-relaxation kinetics taking into account both optical excitation and nonradiative relaxation processes. The probability rates for direct optical transitions from the $h h 1$ states with $m= \pm 3 / 2$ to higher subbands are denoted as $W_{ \pm}$. For linearly polarized light, $W_{+}$and $W_{-}$are equal. For the circular polarization, right-handed, $\sigma_{+}$, or left-handed, $\sigma_{-}$, the rates $W_{ \pm}$are different but, due to time inversion symmetry, satisfy the condition $W_{+}\left(\sigma_{ \pm}\right)=$ $W_{-}\left(\sigma_{\overline{+}}\right)$. If $p_{+}$and $p_{-}$are the $2 \mathrm{D}$ densities of heavy holes with spin $+3 / 2$ and $-3 / 2$, respectively, then the rate equation for $p_{+}$can be written as

$$
\frac{\partial p_{+}}{\partial t}+\frac{p_{+}-p_{-}}{2 \tau_{s}}=-W_{+}+\frac{1}{2}\left(W_{+}+W_{-}\right) .
$$

The corresponding equation for $p_{-}$is obtained by exchange of indices $\pm \rightarrow \mp$. Since the laser pulse duration was longer than any relaxation time we consider the steady-state solution and omit the time derivative in Eq. (1). The second term on the left-hand side of Eq. (1) describes the spin relaxation trying to equalize the polarization of the $\pm 3 / 2$ states. The first term on the right-hand side describes the removal of holes from the $h h 1$ subband due to photoexcitation while the second term characterizes the relaxation of holes which come down to the $+3 / 2$ and $-3 / 2$ states with equal rates (see Fig. 3 insets). The right side of Eq. (1) is proportional to $W_{+}-$ $W_{-}=\frac{\alpha d I}{\hbar \omega}\left(\rho_{0} P_{\text {circ }}-\eta \rho\right)$, where $\rho=\left(p_{+}-p_{-}\right) / p_{s}$ is the hole spin polarization degree, $\rho_{0}$ is the excitation induced spin polarization, and $\eta \approx f_{i} /\left(f_{i}-f_{f}\right) \approx 1$ describes the difference between the population of the initial state, $f_{i}$, and the final state, $f_{f}$, respectively.

Bleaching of absorption with increasing intensity is described by the function $\alpha=\alpha_{0}\left[1+\left(I / I_{s e}\right)\right]^{-1}$, where $\alpha_{0}$ is the absorption coefficient at low intensities and $I_{s e}$ is the characteristic saturation intensity controlled by energy relaxation of the hole gas. Since the photocurrent $j_{\text {LPGE }}$ induced by the linearly polarized radiation is proportional to $\alpha I$, one has

$$
\frac{j_{\mathrm{LPGE}}}{I} \propto \frac{1}{1+\frac{I}{I_{s e}}} .
$$

The photocurrent $j_{\text {CPGE }}$ induced by the circularly polarized radiation is proportional to $W_{+}-W_{-}$. Solving Eqs. (1) and (2) in the steady-state regime, we obtain

$$
\frac{j_{\mathrm{CPGE}}}{I} \propto \frac{1}{1+I\left(\frac{1}{I_{s e}}+\frac{1}{I_{s s}}\right)},
$$

where $I_{s s}=\hbar \omega p_{s} /\left(\alpha_{0} d \tau_{s}\right)$ is the saturation intensity controlled by the hole spin relaxation. The saturation intensities $I_{s s}$ and $I_{s e}$ were extracted from the measured saturation intensities $I_{s}$ of linear and circular photogalvanic current (Fig. 3). The low intensity absorption coefficient was calculated for $\lambda=148 \mu \mathrm{m}$ and takes into account the nonparabolic behavior of the in-plane hole dispersion which differs for different subbands [27]. Using $I_{s s}$ together with 


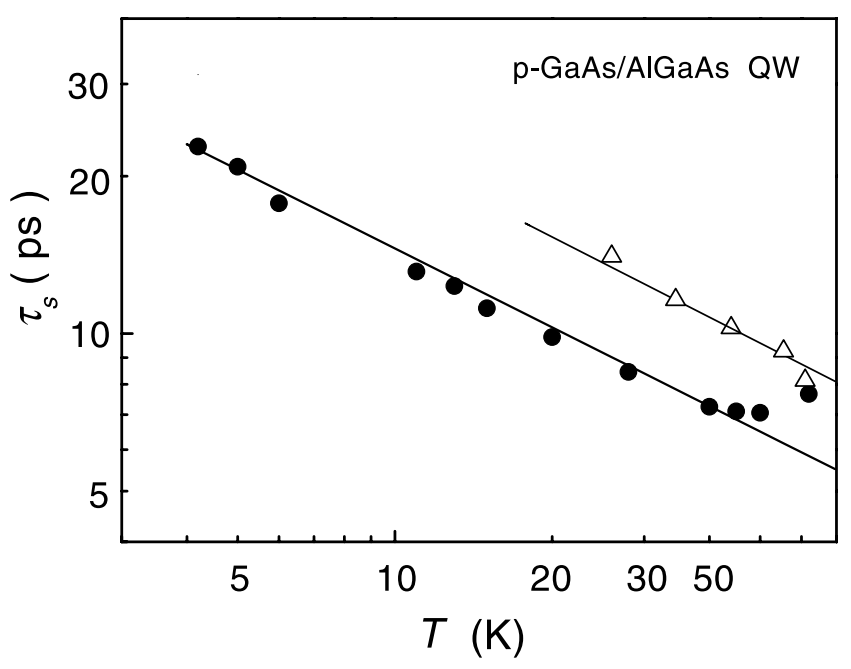

FIG. 4. Experimentally determined spin relaxation times $\tau_{s}$ of holes in $p$-type GaAs/AlGaAs QWs as a function of $T$. Open triangles and full dots correspond to (113)-MBE-grown 15-nm single and multiple (20) QWs, respectively. Free carrier densities of all samples were about $2 \times 10^{11} \mathrm{~cm}^{-2}$ for each QW.

$\alpha_{0}$, spin relaxation times $\tau_{s}$ have been derived. The results are plotted in Fig. 4 as a function of temperature. At low temperatures the relaxation times vary like $T^{-1 / 2}$.

The magnitude of the observed hole spin relaxation time $\tau_{s}$ in $h h 1$ is in agreement with published photoluminescence data $[14-16,29-33]$. There the relation between spin relaxation time and free carrier density has been discussed in terms of the D'yakonov-Perel (DP) and the Bir-Aronov-Pikus (BAP) mechanisms. In our case of monopolar spin orientation no electron-hole was created. Thus, in contrast to all previous experiments using interband excitation, the BAP mechanism is absent and the hole spin relaxation is not affected by high density photocreated carriers, exciton formation, and interband recombination. The DP mechanism was investigated theoretically in $[26,34]$ for spin relaxation of $h h 1$ holes in GaAs based QWs. The values of $\tau_{s}$ on the order of $10 \mathrm{ps}$ as well as the observed temperature dependence $\tau_{s} \propto T^{-1 / 2}$ are in accordance with these calculations for samples with parameters as in our experiment.

In conclusion, our experimental results demonstrate that absorption of terahertz radiation by intervalence band transitions in $p$-type QWs becomes spin sensitive at high power levels. The saturation of circularly polarized radiation yields the spin relaxation times of majority carriers, in our case holes. We emphasize that spin-sensitive bleaching is also expected for intersubband transitions in $n$-type QWs.

We thank L. Golub for helpful discussions. Financial support by the DFG, the RFBR, the INTAS, and the NATO linkage program is gratefully acknowledged.
[1] S. Datta and B. Das, Appl. Phys. Lett. 56, 665 (1990).

[2] G. A. Prinz, Phys. Today 48, No. 4, 58 (1995).

[3] B. E. Kane, Nature (London) 393, 133 (1998).

[4] D. Loss and D. P. DiVincenzo, Phys. Rev. A 57, 120 (1998).

[5] D. P. DiVincenzo et al., Nature (London) 408, 339 (2000).

[6] R. Fiederling et al., Nature (London) 402, 787 (1999).

[7] Y. Ohno et al., Nature (London) 402, 790 (1999).

[8] I. Malajovich et al., Nature (London) 411, 770 (2001).

[9] Optical Orientation, edited by F. Meier and B.P. Zakharchenya (Elsevier Science, Amsterdam, 1984).

[10] S. D. Ganichev et al., Phys. Rev. Lett. 86, 4358 (2001).

[11] J. M. Kikkawa et al., Science 227, 1284 (1997).

[12] D. Hägele et al., Appl. Phys. Lett. 73, 1580 (1998).

[13] J. M. Kikkawa and D. D. Awschalom, Nature (London) 397, 139 (1999).

[14] J. Shah, Ultrafast Spectroscopy of Semiconductor Nanostructures (Springer-Verlag, Berlin, New York, 1999), pp. $243-261$.

[15] L. J. Sham, J. Phys. Condens. Matter 5, A51 (1993).

[16] J. Fabian and S. Das Sarma, J. Vac. Sci. Technol. B 17, 1708 (1999).

[17] E. V. Beregulin et al., Sov. Phys. Semicond. 16, 179 (1982).

[18] C. R. Pidgeon et al., Phys. Rev. Lett. 50, 1309 (1983).

[19] E. V. Beregulin et al., Sov. Phys. Semicond. 21, 615 (1987).

[20] M. Helm et al., Appl. Phys. Lett. 63, 3315 (1993).

[21] W. J. Li et al., Semicond. Sci. Technol. 9, 630 (1994).

[22] S. D. Ganichev et al., Appl. Phys. Lett. 77, 3146 (2000).

[23] Current $j_{x}$ generated by polarized radiation at normal incident was measured as a function of intensity via the voltage drop across a $50 \Omega$ load resistor in a closed circuit. For LPGE linearly polarized radiation with the electric field vector $E$ oriented at $45^{\circ}$ to the $x$ direction was used.

[24] E. L. Ivchenko and G. E. Pikus, Superlattices and Other Heterostructures. Symmetry and Optical Phenomena (Springer, Berlin, 1997).

[25] In the $\Gamma_{8}$-band model based on the isotropic Luttinger Hamiltonian, direct optical transitions between $h h 1$ and $l h 1$ are forbidden at $k=0$. For $k \neq 0$ direct transitions are possible. Band calculations taking into account nonparabolic $h h$ and $l h$ band structure and spectral broadening show that the incident photon energy of $8.3 \mathrm{meV}$ fits the lh1-hh 1 energy separation [26-28]. At the temperatures of this investigation the absorption due to direct optical transitions is much stronger than free carrier absorption caused by indirect transitions [27].

[26] R. Ferreira and G. Bastard, Phys. Rev. B 43, 9687 (1991).

[27] L. E. Vorobjev, D. V. Donetskii, and L. E. Golub, JETP Lett. 63, 977 (1996).

[28] M. Altarelli, U. Ekenberg, and A. Fasolino, Phys. Rev. B 32, 5138 (1985).

[29] T. C. Damen et al., Phys. Rev. Lett. 67, 3432 (1991).

[30] S. Bar-Ad and I. Bar-Joseph, Phys. Rev. Lett. 68, 349 (1992).

[31] B. Baylac et al., Surf. Sci. 326, 161 (1995).

[32] S. Adachi et al., J. Lumin. 72-74, 307 (1997).

[33] M. D. Martin et al., Physica (Amsterdam) 2E, 186 (1998).

[34] G. Bastard and R. Ferreira, Europhys. Lett. 23, 439 (1993). 